\title{
Automated calculation of reduced resistance to a heat transfer of the window based on BIM technologies
}

\author{
Elena Ignatova ${ }^{1, *}$ \\ ${ }^{1}$ Moscow State University of Civil Engineering, Yaroslavskoe shosse, 26, Moscow, 129337, Russia
}

\begin{abstract}
The aim of this work is the development of automated methods of calculation of thermophysical characteristics of the window based on BIM technology. The structure and materials of window frames and glazing are taken into account while calculating reduced resistance to a heat transfer of the window. Software Autodesk Revit was used for creating the information model of the window. As a result of current research a parametric families of windows were created. Each family contains an automatically calculated parameter of reduced resistance to a heat transfer of the window. Windows with one, two and three sashes were considered in the paper. Calculations have been made for wooden and plastic frames. Russian design standards were taken into consideration. The results of the calculations of reduced resistance to a heat transfer of the window are presented in the table at the end of the paper. The introduced method is universal for any window structure. Accurate calculation of heat loss of window units will allow to choose the design decisions and to predict energy costs for building maintenance.
\end{abstract}

\section{Introduction}

Important aspects of green (environmentally friendly) construction are energy-saving and energy-efficiency. In Russia, the production of construction materials and their subsequent transport consume about $8 \%$ of the energy. About 3\% of the energy is consumed during the process of construction. About $90 \%$ of the energy is consumed while operating the object during its entire life cycle. At the same time in Russia operating indicators of energy consumption are about 3 times higher than similar numbers in developed countries with similar natural and climatic conditions [1]. This suggests the need to reduce energy costs while operating the building.

The admission, utilization and loss of the energy are taken into account to calculate the energy balance of the building. The greatest amount of energy is spent for space heating of the building. However, a part of the heat goes into the environment through the outer structure of the building as a result of the process of heat transfer. A significant role in the reduction of heat loss of a building is the choice of structures and materials of windows.

\footnotetext{
*Corresponding author: ignatova@mgsu.ru
} 
Window manufacturers give background information on the resistance to heat transfer of different variants of their glazing and frames. But calculations are needed to get the value of the heat resistance of the entire window. There are interactive programs-calculators for calculating thermophysical characteristics of window, for example [2].

These programs-calculators take into account the dimensions of window units and number of sashes. These programs use the resistance to heat transfer of the frame and of the glazing and calculate the reduced resistance to a heat transfer of the entire window. Window constructions are usually unified in accordance with the standards of manufacturers (Figure $1)$.

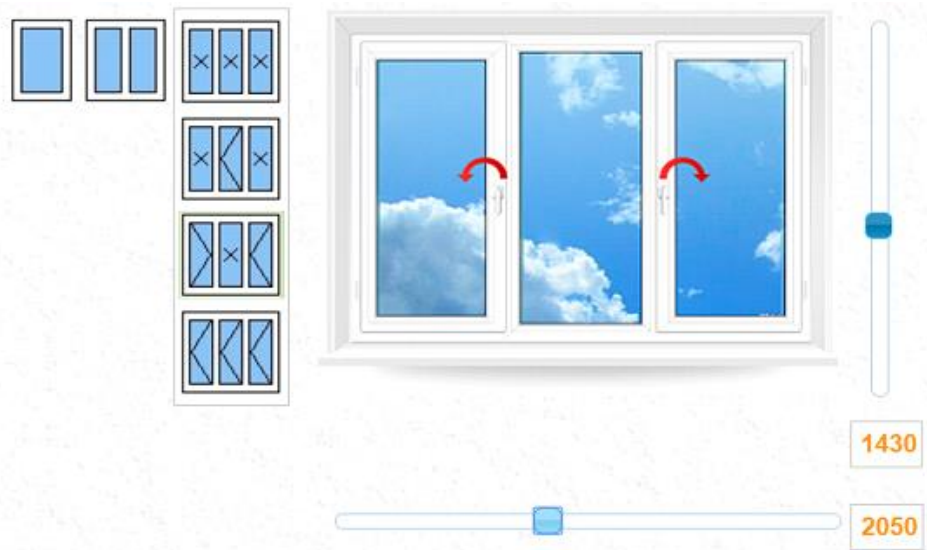

Fig. 1. The choosing of window structure and its size [2].

The reduced resistance to a heat transfer of the whole window $\mathrm{Rw}\left(\mathrm{m} 2 \cdot{ }^{\circ} \mathrm{C} / \mathrm{W}\right)$ can be calculated using the following formula:

$$
R w=\frac{\sum_{i} F i}{\sum_{i} \frac{F i}{R i}},
$$

where $\mathrm{Fi}$ - area of the $\mathrm{i}$-th uniform zone, $\mathrm{m}^{2}$;

$\mathrm{Ri}$ - resistance to a heat transfer of the $\mathrm{i}$-th uniform zone, $\mathrm{m}^{2} \cdot{ }^{\circ} \mathrm{C} / \mathrm{W}$.

During the research the assumption was made that the exact calculation of the thermal characteristics of windows at the construction stage can reduce heat loss in the building and can increase its efficiency. In addition, analysis of heat loss for various options of glazing should be implemented by the automated method.

The modern development of automated design has led to the use of building information modeling (BIM) technology. The most common software for building information modeling is Autodesk Revit [3]. This program has the function of calculating the energy efficiency of a building based on its information model [4]. However, the calculation process uses the cloud service and private algorithms, and there are no intermediate data calculations. Windows from the standard Autodesk Revit library are editable parametric objects, and these parameters are length, width, height and material. Due to the small number of parameters these families are poorly adapted to many design decisions.

The purpose of this research was to develop an automated method of calculation of reduced resistance to a heat transfer of windows in the building information modeling software Autodesk Revit.

As a result of research a parametric family of models of the window was developed. This family allows to compute thermophysical characteristics for calculating the energy efficiency of the building. 


\section{Literature review}

Approaches to solving the problem of calculating the heat loss of the building elements was considered in lots of papers. Paper [5] describes the results of numerical investigation of heat transfer resistance of enclosing structures based on Ansys program. Paper [6] investigates the effect of wind speed on the change of heat transfer resistance of translucent structures of high buildings. Paper [7] has developed a methodology and program for calculating heat loss of a wall with respect to windows. Paper [8] considers various activities to increase given thermal resistance of the window. They also analyze the value of the reduced resistance to heat transfer of windows of different manufactures. Paper [9] investigates the dependence of thermal resistance to heat transfer of the window on the tilt angle, the geometric dimensions of the glazing, selective coating of glass and the physical characteristics of the gas filling the space between glass panes.

In Russia, the reduced resistance to a heat transfer $\mathrm{R}\left(\mathrm{m} 2 \cdot{ }^{\circ} \mathrm{C} / \mathrm{W}\right)$ is used for evaluating the thermal protective characteristics of structures. Minimal regulatory requirements for thermal resistance of windows are defined in GOST 26602.1-99 [10]. Tables of experimental data on the resistance to heat transfer of different window structures are presented in GOST 23166-99 [11].

The current research is based on the theory of calculating the heat loss of the building and on the theory of building information modeling with the implementation of software Autodesk Revit.

In Russia the building information modeling (BIM) technology is discussed and used by enthusiasts for over a decade. In 2014, the Russian government established a mandatory order of BIM application [12]. This event gave impulse to the widespread of BIM implementation in design and construction organizations. The building information modeling technology allows to store not only geometric characteristics of the building elements but also any other information, including physical characteristics [13]. On the base of available information it is possible to solve many engineering problems in the area of design, calculations, analysis and model evaluation $[14,15,16,17]$.

The activities with building information model in Autodesk Revit are based on the creation and application of parametric families of elements of the building. The parameters, their values, or necessary relations are set for each element of the building. Parameters can be specified by analytical expressions. The change of one parameter of the structural element will automatically lead to the change of other related parameters. Thus, various options of structural elements can be obtained on the basis of a single parametric family. Software Autodesk Revit allows to combine several simple families into more complex composite family $[18,19,20]$. The creation of the family of multi-sash window is based on this principle.

A parent family of the window consists of the family of frames and several variants of sash families. In turn, the parametric family of the sash consists of interconnected collections of glazing and frame (Figure 2).
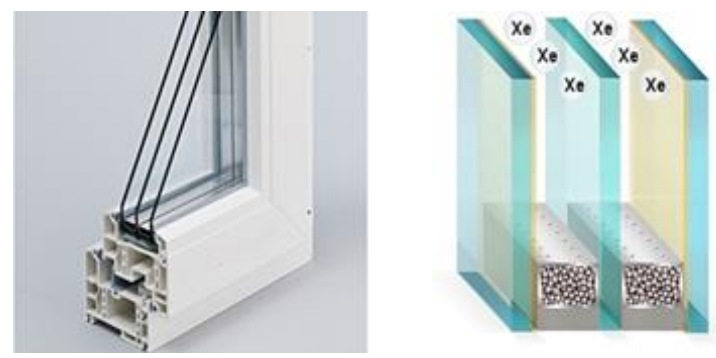

Fig. 2. The parts of sash. 


\section{Materials and methods}

The real reduced resistance to a heat transfer of the window is determined on the base of the results of certification tests. In their absence, reduced resistance can be calculated.

The development of an automated method of calculation of reduced resistance to a heat transfer of windows was held in the building information modeling software Autodesk Revit 2016. The construction of window was developed on the base of standard materials for windows [10]. For this purpose the set of collections of glazing and window frames was developed. Each family of glazing or window frame has a material parameter that can be modified depending on the structure of the window. The material has graphic, strength and thermophysical characteristics, which can be used to render the window model or to calculate other necessary characteristics of the family.

Glazing may consist of a single glass, two or three glasses. Type of the glass and glass thickness correspond industry standards. There exist windows with gas filling the internal volume. Theoretically, the number, type, thickness of glass, the distance between the panes and the gas between the panes can be used to calculate the resistance to heat transfer of the glazing. However, the characteristics of resistance to heat transfer are already known for all real types of glazing, and these characteristics are experimentally confirmed.

The case with the thermal characteristics of the frame is similar. Window frames may be plastic or wooden. There are many options of window frames. In our paper we assume that the frames of all window sashes are the same. For a sash of the window the resistance to heat transfer and the heat loss depend on the size of the sash. To be more accurate, they depend on area ratio of the glazing and the entire window. For any size of sash the calculation of the resistance to heat transfer and the heat loss is carried out automatically. In the case of complex compound family, that consists of the frame family and one of the several sash families, the calculation of thermal characteristics of the entire window is carried out automatically and takes into account the construction, proportions, materials and thermal properties of nested families. The general scheme of the window elements is shown in Figure 3.
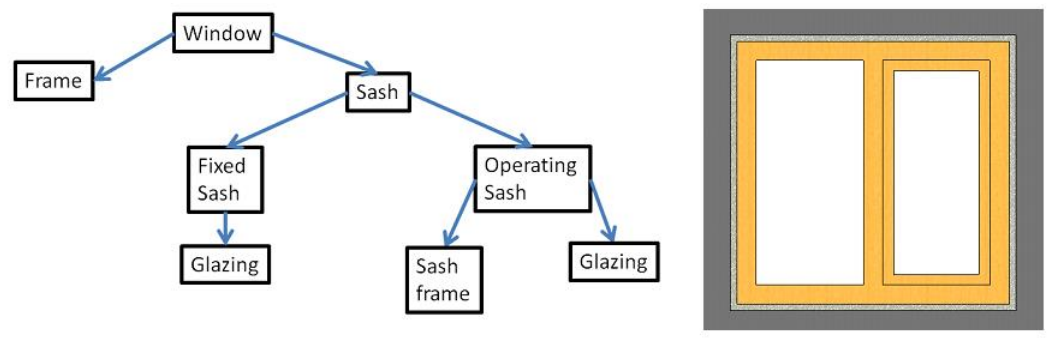

Fig. 3. The elements of window family.

The reduced resistance to a heat transfer of an operating sash $R s\left(m 2 \cdot{ }^{\circ} \mathrm{C} / \mathrm{W}\right)$ can be calculated using the following formula:

$$
R s=\frac{F g+F f}{\frac{F g}{R g}+\frac{F f}{R f}},
$$

where $\mathrm{Fg}$ - area of the glazing, $\mathrm{m}^{2}$;

$R g$ - resistance to a heat transfer of the glazing, $\mathrm{m}^{2} \cdot{ }^{\circ} \mathrm{C} / \mathrm{W}$;

$F f$ - area of the frame, $\mathrm{m}^{2}$;

$R f$ - resistance to a heat transfer of the frame, $\mathrm{m}^{2} \cdot{ }^{\circ} \mathrm{C} / \mathrm{W}$;

The reduced resistance to a heat transfer of entire window $R w$ is calculated in a similar way. 


\section{Results}

The suggested method of modeling of the window and calculating of its reduced resistance to heat transfer was implemented for windows of standard structures that are common in Russia (Figure 4).
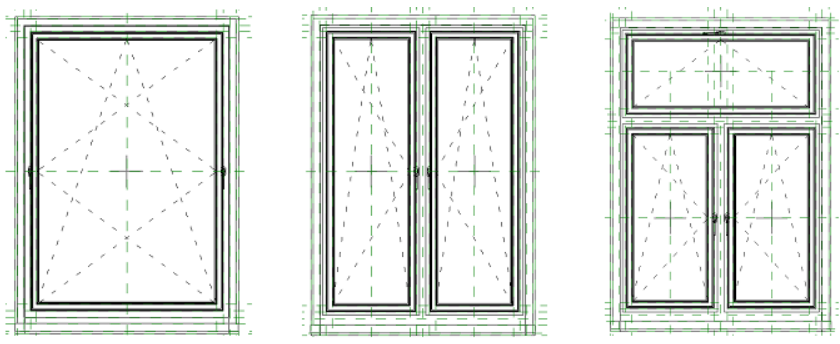

Fig. 4. Models of windows with one, two and three sashes.

It is possible to choose the direction of the sashes. The change of the direction leads to the change of the location of the handle and the symbols of opening the window. If the fixed sash is selected, the glazing is inserted into the window frame. The frame of the sash is parametrically related to the size of the window frame.

The glazing consists of the set of elements, such as glass pane, spacer, desiccant, sealants, filling gas. The dimensions of the glazing are parametric and are associated with the size of the sash (Figure 5).
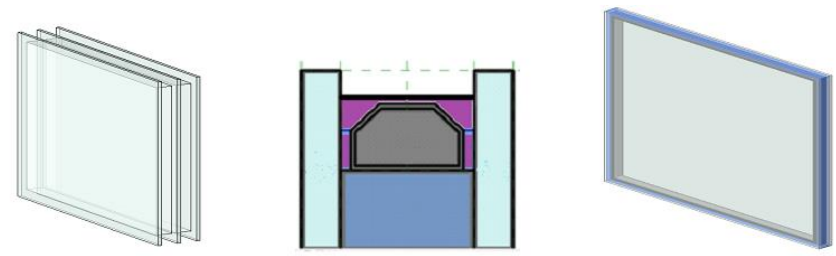

Fig. 5. The structure of glazing.

Different kinds of wooden and plastic windows were considered. The results are shown in the table 1.

Table 1. Rw of different windows.

\begin{tabular}{|c|c|c|c|c|c|c|}
\hline $\begin{array}{c}\text { Number } \\
\text { of sashes }\end{array}$ & $\begin{array}{c}\text { Window } \\
\text { size }\end{array}$ & Glazing & Frame & Rg & Rf & Rw \\
\hline 1 & $1400 \times 2000$ & 4M1-8-4M1 & FloraIV-78 Fir & 0.28 & 0.71 & 0.334 \\
\hline 1 & $1400 \times 2000$ & 4M1-Ar8-4M1 & FloraIV-78 Fir & 0.30 & 0.71 & 0.355 \\
\hline 1 & $1400 \times 2000$ & 4M1-8-4M1 & FloraIV-78 Pine & 0.28 & 0.43 & 0.309 \\
\hline 1 & $1400 \times 2000$ & 4M1-Ar8-4M1 & FloraIV-78 Pine & 0.30 & 0.43 & 0.327 \\
\hline 2 & $1400 \times 2000$ & 4M1-8-4M1 & FloraIV-78 Pine & 0.28 & 0.43 & 0.313 \\
\hline 2 & $1400 \times 2000$ & 4M1-Ar8-4M1 & FloraIV-78 Pine & 0.30 & 0.43 & 0.330 \\
\hline 2 & $1200 \times 1200$ & 4M1-16-4M1 & Veka Softline-82/7 & 0.32 & 1.06 & 0.448 \\
\hline 2 & $1200 \times 1200$ & 4M1-Ar16- & Veka Softline-82/7 & 0.34 & 1.06 & 0.471 \\
\hline 3 & $1400 \times 2000$ & 4M1-8-4M1 & FloraIV-78 Fir & 0.28 & 0.71 & 0.352 \\
\hline 3 & $1400 \times 2000$ & 4M1-Ar8-4M1 & FloraIV-78 Fir & 0.30 & 0.71 & 0.372 \\
\hline
\end{tabular}




\section{Discussion}

Automated calculation of the reduced resistance to a heat transfer of the window can be done in different ways, including the usage of programs-calculators. In that case, afterwards the data has to be transferred to other calculation programs manually. The building information modelling technology implies the activity with a common model of the building. Model analysis can also be carried out by means of the building information modeling software. Therefore, internal calculation of the physical characteristics of the window in Autodesk Revit is justified.

It should be understood that the calculation of the reduced resistance to a heat transfer of the window is only the first step in determining the heat loss of a building to calculate its energy efficiency. For further calculations it is necessary to know the construction region and the climatic characteristics. To design an energy-efficient building it is necessary to know the minimum possible characteristics of resistance to heat transfer of building structures.

It is convenient to calculate the heat loss through windows in Autodesk Revit inside the specification tables of the windows. Tabular view of information allows to add extra fields (columns) to calculate the heat loss through the windows. It is also possible to summarize the heat loss through all windows. The information can be sorted by the floors, window types, etc. The method of creating window model with the calculation of the thermophysical characteristics is universal and can be used for windows of various constructions.

In the research window models with one, two and three sashes were created. A problem of creating a model of universal window was discussed. That is the model, from which a broad variety of other windows could be obtained. However, a large number of conditions are imposed on the model, and a large number of control parameters start to degrade the performance of the designer. If the building has a large number of windows, the computer operates with building information model much slower.

There was a discussion about the level of detalization of the window model. Is it important for determining the heat loss through the window to create a geometric representation of all parts of the glazing and the frame? Is it possible to automatically calculate the resistance to a heat transfer of the glazing on the base of the geometrical and physical characteristics of its parts?

\section{Conclusions}

This research focuses on the particular problem of computing the heat loss through the windows for solving the problem of energy efficiency of the building. The method of automated calculation of reduced resistance to a heat transfer of the window was proposed. The method was implemented by the means of the building information modeling software Autodesk Revit. During the research the hierarchy of Autodesk Revit families was established for organizing the composite window family. The relationships with geometric and thermophysical parameters of the families were defined. In the composite family the automatic calculation of reduced resistance to heat transfer of the entire window was organized. The method of further calculation of the heat loss through the windows was proposed. For the additional verification of the window model it is planned to search for the results of the experimental studies for which all of the design properties are known.

\section{References}

1. P. Petronijević, N. Ivanišević, M. Rakočević, D. Arizanović, Journal of Applied Engineering Science 1(10), 43-48 (2012) ISSN 1451-4117, doi:10.5937/jaes10-1664 
2. S.S. Golubev, Scientific and Technical Volga region Bulletin 5, 93-97 (2011)

3. Y.A. Eldashov, S.G. Sesunin, V.N. Kovrov, Vestnik MGSU 3, 146-149 (2009)

4. V.M. Tusnina, A.R. Tusnin, Vestnik MGSU 1-2, 123-128 (2011)

5. E.S. Konisheva, E.V. Korepanov, Energy and resource saving in industry, housing and communal services and agriculture, 119-125 (2016)

6. E.V. Korepanov, Mathematical modeling of heat transfer through the exterior walls of buildings with windows (Kalashnikov Izhevsk State Technical University, Izhevsk, 2011)

7. A. Zinatullin, E. Chibisova, Strategy of sustainable development of regions in Russia 5, 324-328 (2011)

8. V.V. Talapov, Basis of BIM: introduction to building information modeling (DMK Press, Moscow, 2011)

9. E.V. Ignatova, Abstracts of the 14th International Conference on Computing in Civil and Building Engineering Moscow, 132-133 (2012)

10. E.V. Ignatova, Vestnik MGSU 9, 241-246 (2012)

11. E.V. Ignatova, Advances in Energy Science and Equipment Engineering II, Taylor \& Francis Group, 653-657 (2017)

12. E. Ignatova, H. Kirschke, E. Tauscher, K. Smarsly, Proceedings of the 20th International Conference on the Applications of Computer Science and Mathematics in Architecture and Civil Engineering, 68-75 (2015)

13. A.L. Lanzov, AutoDesk Revit 2015. Computer aided design of buildings (RIOR Press, 2014)

14. J. Vandezande, E. Krygiel, Mastering Autodesk. Revit architecture 2016 (SYBEX, 2016) 